\title{
Parameter Estimation in a Three-dimensional Wind Field Model Using Genetic Algorithms
}

\author{
Eduardo Rodríguez, Gustavo Montero, Rafael Montenegro, \\ José María Escobar, and José María González-Yuste \\ University Institute of Intelligent Systems and Numerical Applications in Engineering, \\ University of Las Palmas de Gran Canaria, \\ Edificio Instituto Polivalente, Campus Universitario de Tafira, \\ 35017 Las Palmas de Gran Canaria, Spain \\ barrera@dma.ulpgc.es, \{gustavo, rafa\}@dma.ulpgc.es, \\ escobar@cic.teleco.ulpgc.es, josem@sinf.ulpgc.es
}

\begin{abstract}
The efficiency of a mass consistent model for wind field adjustment depends on several parameters that arise in various stages of the process. First, those involved in the construction of the initial wind field using horizontal interpolation and vertical extrapolation of the wind measures registered at meteorological stations. On the other hand, the Gauss precision moduli which allow from a strictly horizontal wind adjustment to a pure vertical one. In general, the values of all of these parameters are based on empirical laws. The main goal of this work is the estimation of these parameters using genetic algorithms, such that the wind velocities observed at the measurement station are regenerated as much as possible by the model.
\end{abstract}

\section{Introduction}

Mass consistent models are diagnostic models for constructing wind velocity fields from a few experimental measurements. In general, these models are defined by the physical laws of an incompressible fluid, by the empirical design of the wind profiles and by the values of velocities measured at the stations. This explains the existence of many parameters in the model. Some of them are clearly bounded and defined, while others are still under discussion and interpretation. Our work deals with the latter ones. There are many methods for the resolution of inverse problems involving parameter estimation and they have been largely studied in the literature. Among them, we have chosen a robust and flexible tool: genetic algorithms, which allow to solve linear and non-linear multiparameter optimisation problems.

This work has been structured as follows. First, the wind model is summarised in Sect. 2. We remark the studied parameters in Sect. 3. Next, in Sect. 4, the fitness function is established and genetic algorithms are briefly introduced, with their properties and possibilities used in this work. Numerical experiments are shown in Sect. 5 and, finally, conclusions are presented in Sect. 6 .

^ Partially supported by MCYT, Spain. Grant contract: REN2001-0925-C03-02/CLI 


\section{Mass Consistent Model in 3-D}

This model [14] is based on the continuity equation for an incompressible flow with constant air density in the domain $\Omega$ and no-flow-through conditions on $\Gamma_{b}$

$$
\begin{array}{ll}
\boldsymbol{\nabla} \cdot \boldsymbol{u}=0 & \text { in } \Omega . \\
\boldsymbol{n} \cdot \boldsymbol{u}=0 & \text { on } \Gamma_{b} .
\end{array}
$$

We formulate a least-square problem in $\Omega$ with $\boldsymbol{u}(\widetilde{u}, \widetilde{v}, \widetilde{w})$ to be adjusted

$$
E(\boldsymbol{u})=\int_{\Omega}\left[\alpha_{1}^{2}\left(\left(\widetilde{u}-u_{0}\right)^{2}+\left(\widetilde{v}-v_{0}\right)^{2}\right)+\alpha_{2}^{2}\left(\widetilde{w}-w_{0}\right)^{2}\right] d \Omega .
$$

where the interpolated wind $\boldsymbol{v}_{0}=\left(u_{0}, v_{0}, w_{0}\right)$ is obtained from experimental measurements, and $\alpha_{1}, \alpha_{2}$ are the Gauss precision moduli. This problem is equivalent to find a saddle point $(\boldsymbol{v}, \phi)$ of the Lagrangian (see [27])

$$
E(\boldsymbol{v})=\min _{\boldsymbol{u} \in K}\left[E(\boldsymbol{u})+\int_{\Omega} \phi \boldsymbol{\nabla} \cdot \boldsymbol{u} d \Omega\right] .
$$

being $\boldsymbol{v}=(u, v, w), \phi$ the Lagrange multiplier and $K$ the set of admissible functions. The Lagrange multipliers technique is used to minimise the problem (4), whose minimum comes to form the Euler-Lagrange equations

$$
u=u_{0}+T_{h} \frac{\partial \phi}{\partial x}, \quad v=v_{0}+T_{h} \frac{\partial \phi}{\partial y}, \quad w=w_{0}+T_{v} \frac{\partial \phi}{\partial z} .
$$

where $T=\left(T_{h}, T_{h}, T_{v}\right)$ is the diagonal transmissivity tensor, with $T_{h}=\frac{1}{2 \alpha_{1}^{2}}$ and $T_{v}=\frac{1}{2 \alpha_{2}^{2}}$. Since $\alpha_{1}$ and $\alpha_{2}$ are constant in $\Omega$, the variational approach results in an elliptic problem substituting (5) in (1)

$$
\frac{\partial^{2} \phi}{\partial x^{2}}+\frac{\partial^{2} \phi}{\partial y^{2}}+\frac{T_{v}}{T_{h}} \frac{\partial^{2} \phi}{\partial z^{2}}=-\frac{1}{T_{h}}\left(\frac{\partial u_{0}}{\partial x}+\frac{\partial v_{0}}{\partial y}+\frac{\partial w_{0}}{\partial z}\right) \text { in } \Omega .
$$

The boundary conditions result as follows (Dirichlet condition for open or flow-through boundaries and Neumann condition for terrain and top)

$$
\begin{aligned}
\phi & =0 \text { on } \Gamma_{a} . \\
\boldsymbol{n} \cdot T \nabla \mu & =-\boldsymbol{n} \cdot \boldsymbol{v}_{0} \text { on } \Gamma_{b} .
\end{aligned}
$$

The classical formulation of the problem given by (6)-(8), is discretized using a tetrahedral mesh of finite elements (see [12]) that leads to a set of $4 \times 4$ elemental matrices and $4 \times 1$ elemental vectors, which are assembled into a global linear system of equations. A preconditioned conjugate gradient method is used for solving this symmetric linear system. 


\subsection{Horizontal Interpolation}

The wind speeds measured at station height $z_{m}$ are interpolated in function of the distance and the height difference between each point and the station [14]

$$
\boldsymbol{v}_{0}\left(z_{m}\right)=\varepsilon \frac{\sum_{n=1}^{N} \frac{\boldsymbol{v}_{n}}{d_{n}^{2}}}{\sum_{n=1}^{N} \frac{1}{d_{n}^{2}}}+(1-\varepsilon) \frac{\sum_{n=1}^{N} \frac{\boldsymbol{v}_{n}}{\left|\Delta h_{n}\right|}}{\sum_{n=1}^{N} \frac{1}{\left|\Delta h_{n}\right|}} .
$$

where $\boldsymbol{v}_{n}$ is the velocity observed at station $n, N$ is the number of stations considered in the interpolation, $d_{n}$ is the horizontal distance from station $n$ to the point where we are computing the wind velocity, $\left|\Delta h_{n}\right|$ is the height difference between station $n$ and the studied point, and $\varepsilon$ is a weighting parameter $(0 \leq \varepsilon \leq$ 1 ), which allows to give more importance to one of these interpolation criteria.

\subsection{Vertical Profile of Wind}

We have considered a logarithmic profile in the surface layer, which takes into account the previous horizontal interpolation, as well as the effect of roughness and the air stability (neutral, stable or unstable atmosphere, according to the Pasquill stability class) on the wind intensity and direction. Above the surface layer, a linear interpolation is carried out using the geostrophic wind. The logarithmic profile is given by

$$
\boldsymbol{v}_{0}(z)=\frac{\boldsymbol{v}^{*}}{k}\left(\log \frac{z}{z_{0}}-\Phi_{m}\right) \quad z_{0}<z \leq z_{s l} .
$$

where $\boldsymbol{v}^{*}$ is the friction velocity, $k$ is von Karman constant, $z_{0}$ is the roughness length and $z_{s l}$ is the height of the surface layer. Function $\Phi_{m}$ depends on the air stability

$$
\begin{array}{cc}
\Phi_{m}=0 & \text { (neutral) } . \\
\Phi_{m}=-5 \frac{z}{L} & \text { (stable) } . \\
\Phi_{m}=\log \left[\left(\frac{\theta^{2}+1}{2}\right)\left(\frac{\theta+1}{2}\right)^{2}\right]-2 \arctan \theta+\frac{\pi}{2} & \text { (unstable) } .
\end{array}
$$

where $\theta=\left(1-16 \frac{z}{L}\right)^{1 / 4}$ and $\frac{1}{L}=a z_{0}^{b}$, with $a, b$, depending on the Pasquill stability class. $L$ is the so called Monin-Obukhov length. The friction velocity is obtained at each point from the interpolated measurements at the height of the stations (horizontal interpolation)

$$
\boldsymbol{v}^{*}=\frac{k \boldsymbol{v}_{0}\left(z_{m}\right)}{\log \frac{z_{m}}{z_{0}}-\Phi_{m}} .
$$


The height of the planetary boundary layer $z_{p b l}$ above the ground is chosen such that the wind intensity and direction are constant at that height

$$
z_{p b l}=\frac{\gamma\left|\boldsymbol{v}^{*}\right|}{f}
$$

where $f=2 \omega \sin \varphi$ is the Coriolis parameter ( $\omega$ is the earth rotation and $\varphi$ the latitude), and $\gamma$ is a parameter depending on the atmospheric stability. The mixing height $h$ is considered to be equal to $z_{p b l}$ in neutral and unstable conditions. In stable conditions, Zilitinkevich suggested (see [3])

$$
h=\gamma^{\prime} \sqrt{\frac{\left|\boldsymbol{v}^{*}\right| L}{f}} .
$$

where $\gamma^{\prime}$ is a constant of proportionality. The height of surface layer is $z_{s l}=\frac{h}{10}$. From $z_{s l}$ to $z_{p b l}$, a linear interpolation with geostrophic wind $\boldsymbol{v}_{g}$ is carried out

$$
\begin{aligned}
\boldsymbol{v}_{0}(z) & =\rho(z) \boldsymbol{v}_{0}\left(z_{s l}\right)+[1-\rho(z)] \boldsymbol{v}_{g} \quad z_{s l}<z \leq z_{p b l} . \\
\rho(z) & =1-\left(\frac{z-z_{s l}}{z_{p b l}-z_{s l}}\right)^{2}\left(3-2 \frac{z-z_{s l}}{z_{p b l}-z_{s l}}\right) .
\end{aligned}
$$

Finally, this model assumes $\boldsymbol{v}_{0}(z)=\boldsymbol{v}_{g}$ if $z>z_{p b l}$ and $\boldsymbol{v}_{0}(z)=0$ if $z \leq z_{0}$.

\section{Discussion on the Parameters to Be Estimated}

First, we will consider the so called stability parameter

$$
\alpha=\frac{\alpha_{1}}{\alpha_{2}}=\sqrt{\frac{T_{v}}{T_{h}}} .
$$

since the minimum of the functional given by (3) is the same if we divide it by $\alpha_{2}^{2}$. On the other hand, for $\alpha>>1$ flow adjustment in the vertical direction predominates, while for $\alpha<<1$ flow adjustment occurs primarily in the horizontal plane. Thus, the selection of $\alpha$ allows the air to go over a terrain barrier or around it, respectively [18]. Moreover, the behaviour of mass consistent models in many numerical experiments has shown that they are very sensitive to the values chosen for $\alpha$. Therefore, we shall give particular attention to this problem. In the past, many authors have studied the parametrisation of stability, since the difficulty in determining the correct values of $\alpha$ have limited the possible wide use of mass-consistent models in complex terrain. Sherman [21], Kitada et al. [9] and Davis et al. [4], proposed to take $\alpha=10^{-2}$, i.e., proportional to the magnitude of $w / u$. Other authors such as Ross et al. [20] and Moussiopoulos et al. [16] related $\alpha$ to the Froude number. Geai [7], Lalas et al. [10] and Tombrou et al. [24], make the $\alpha$ parameter vary in the vertical direction. Finally, Barnard et al. [2] proposed a procedure to obtain $\alpha$ for each single wind field simulation. The main idea is to use $N$ observed wind speeds to obtain the wind field and to 
keep the rest, $N_{r}$, as a reference. Then, several simulations are performed with different values of $\alpha$. The value which gives the best agreement with the reference observations is taken to be the final magnitude of the stability parameter. Since this method provides values of $\alpha$ that are only reliable for each particular case, it cannot provide an a priori value suitable for other simulations. Here, we follow a version of the method proposed in [2], using genetic algorithms as optimisation technique which lead to an automatic selection of $\alpha$.

The second parameter to be estimate is the weighting coefficient $\varepsilon(0 \leq \varepsilon \leq 1)$ of (9). Note that $\varepsilon \rightarrow 1$ signifies more importance of the horizontal distance from each point to the measurement stations, while $\varepsilon \rightarrow 0$ signifies more importance of the height difference between each point and the measurement stations [14]. In general, the second approach has been used for complex terrains. On the other hand, the first approach has been widely used for problems with regular topography or in 2-D horizontal analysis. In realistic applications, the possibility of existing zones with complex orography and others with regular one, suggests that an intermediate value of $\varepsilon$ should be more suitable.

The next parameter to discuss is $\gamma$, given in (13) and related to the height of the planetary boundary layer. There exist different versions of where to search this parameter. Panofsky et al. [17] proposed the interval $[0.15,0.25]$. On the other hand, Ratto [19] directly suggested $\gamma=0.3$ in the WINDS code, while $\gamma$ is located in $[0.3,0.4]$ by de Baas [1]. Therefore, in our simulations, the search space for $\gamma$ must include all these possibilities.

Finally, we are interested in obtaining suitable values of the parameter $\gamma^{\prime}$ involved in the computation of the mixing height for stable atmosphere, see (14). Garratt [6] proposed $\gamma^{\prime}=0.4$. Also in the WINDS code one may find bounds of $\gamma^{\prime}$ around 0.4. Thus, the value of $\gamma^{\prime}$ will be searched in the surroundings of 0.4.

\section{Genetic Algorithms}

Genetic algorithms (GAs) are optimisation tools based on the natural evolution mechanism. They produce successive trials that have an increasing probability to obtain a global optimum. This work is based on the model developed by Levine [11]. The most important aspects of GAs are the construction of an initial population, the evaluation of each individual in the fitness function, the selection of the parents of the next generation, the crossover of those parents to create the children, and the mutation to increase diversity.

Two population replacements are commonly used. The first, the generational replacement, replaces the entire population each generation [8]. The second, known as steady-state, only replaces a few individuals each generation $[23,25$, 26]. Stopping criteria are iteration limit exceeded, population too similar, and no change in the best solution found in a given number of iterations. Initial population is randomly generated.

The selection phase allocates an intermediate population on the basis of the evaluation of the fitness function. We have considered four selection schemes 
[11]: proportional selection (P), stochastic universal selection (SU), binary tournament selection (BT) and probabilistic binary tournament selection (PBT).

The crossover operator takes bits from each parent and combines them to create a child. One-point (OP) and uniform (U) crossover operators are used here. The first one selects randomly the place where each of the parents strings are broken in two substrings. Children will be the union of first substring of one parent and the second of the other. Uniform crossover depends on the probability of exchange between two bits of the parents [22].

The mutation operator is better used after crossover [5]. It allows to reach individuals on the search space that could not be evaluated otherwise. When part of a chromosome has been randomly selected to be mutated, the corresponding genes belonging to that part are changed. This happens with probability $p$. This work deals with four mutation operators. Three of them are of the form $\nu \leftarrow \nu \pm p \times \nu$, where $\nu$ is the existing allele value, and $p$ may be a constant value (C), chosen uniformly from the interval $(0, \beta)$ with $\beta \leq 1$ (U), or selected from a Gaussian distribution $(\mathrm{G})$. The fourth operator $(\mathrm{R})$ simply replaces $\nu$ with a value selected uniformly random from the initialisation range of that gene.

The fitness function plays the role of the environment. It evaluates each string of a population. This is a measure, relative to the rest of the population, of how well that string satisfies a problem-specific metric. The values are mapped to a nonnegative and monotonically increasing fitness value. In the numerical experiments with this wind model, we look for suitable values of $\alpha, \varepsilon, \gamma$ and $\gamma^{\prime}$. For this purpose, the average relative error of the wind velocities given by the model with respect to the measures at the reference stations is minimised

$$
F\left(\alpha, \varepsilon, \gamma, \gamma^{\prime}\right)=\frac{\sum_{n=1}^{N_{r}} \frac{\left|\boldsymbol{v}_{n}-\boldsymbol{v}\left(x_{n}, y_{n}, z_{n}\right)\right|}{\left|\boldsymbol{v}_{n}\right|}}{N_{r}} .
$$

where $\boldsymbol{v}\left(x_{n}, y_{n}, z_{n}\right)$ is the wind velocity obtained by the model at the location of station $n$, and $N_{r}$ is the number of reference stations.

\section{$5 \quad$ Numerical Experiments}

We consider the same wind field problem related to the southern area of La Palma Island (Canary Islands) which was defined in [15]. A $45600 \times 31200 \times 9000 \mathrm{~m}^{3}$ domain with real data of the topography is discretized using the code developed in [12]. The maximum height in this zone of the island is $2279 \mathrm{~m}$. The mesh contains 11578 nodes and 52945 tetrahedra; see Fig. 1. The wind measurements were taken in four stations: MBI, MBII, MBIII and LPA. From the three cases studied in [15], we have selected case I with softly unstable conditions and case III with softly stable conditions in order to test the procedure for different stability conditions of the atmosphere. Due to the small number of available data, we have used the observed wind speeds of stations MBI, MBII and LPA to obtain the interpolated wind field (9), i.e., $N=3$, and the measurement of MBIII is considered as reference station in the fitness function (18), i.e., $N_{r}=1$. 


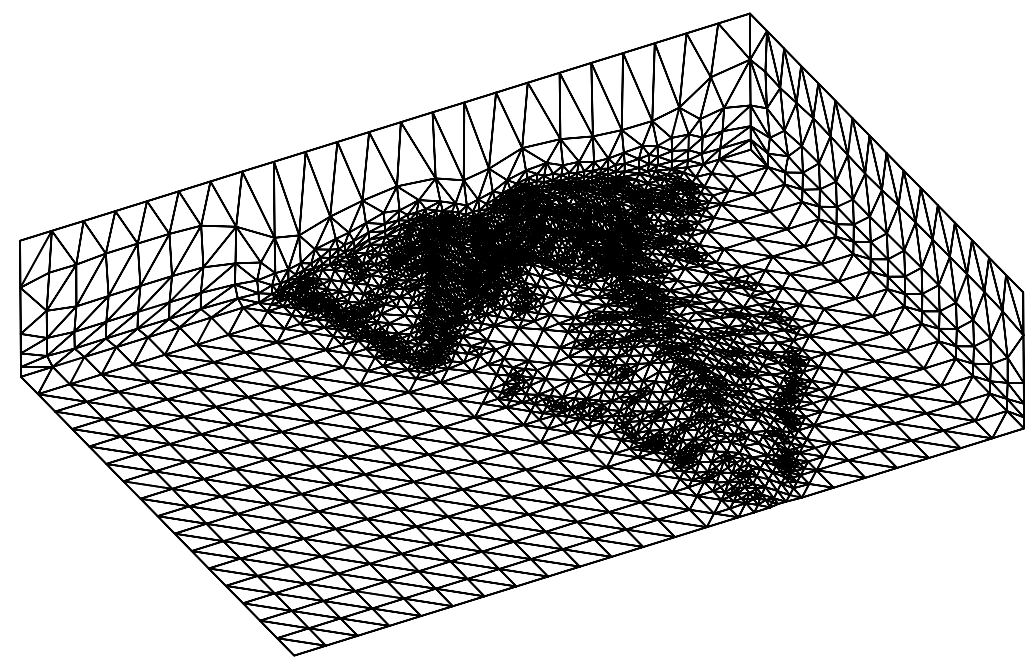

Fig. 1. Detail of the finite element mesh used for the numerical experiment. We only plot the triangulation of the terrain and two vertical wall in order to hold clarity

In the first application (case I), the parameter $\gamma^{\prime}$ is not involved in the modelling due to the unstable condition of the atmosphere, i.e., $h=z_{p b l}$. Thus, only $\alpha, \varepsilon$ and $\gamma$ will be estimated in this case. The experiment has been divided in two stages. First, we fix $\gamma=0.3$ and estimate $\alpha \in\left[10^{-3}, 10\right]$ and $\varepsilon \in[0,1]$. The second column of Tab. 1 (Stage 1) shows the values obtained for $\alpha$ and $\varepsilon$, which suggest a nearly vertical wind adjustment and remark the complexity of the terrain respectively. Note that we obtain with the model an error at station MBIII about $10.7 \%$. The strategy of GAs $(B T, U, R)$ corresponds to the most efficient selection, crossover and mutation operators after several tests with different combinations. In the second stage, $\alpha, \varepsilon$ and $\gamma \in[0.15,0.5]$ are estimated. The results are also shown in the third column of Tab. 1. We observe that $\alpha$ is near the maximum value of the space of search, $\varepsilon$ remains quite small and $\gamma$ is reduced, such that the error at station MBIII is $10.7 \%$. We remark that in this experiment the worst evaluation of the fitness function, corresponding to values of the parameters in the search space, yields an error of $72.19 \%$. Therefore, the knowledge of the studied parameters is essential for the efficiency of the numerical model.

For the second experiment (case III) we have followed a similar procedure. Now, $\gamma^{\prime} \in[0.15,0.5]$ must be also considered. First, a problem with two unknown parameters $(\alpha, \varepsilon)$ is solved. The second column of Tab. 2 (Stage 1) shows the values obtained for $\alpha, \varepsilon$. Next, four problems arising from fixing one of the parameters each time, respectively, are studied (Stages 2-5). Finally, the four 
parameters are estimated at the same time in Stage 6. The atmospheric stable conditions reduce the vertical adjustment predominance arising in the previous experiment with unstable conditions, as well as augment the importance of the horizontal distance in the interpolation of the observed wind speeds. The minimum error obtained at station MBIII was about $22.2 \%$, while the error related to the worst evaluation was $118.04 \%$.

In both experiments, the number of individuals of the initial population was 100, except for stage 6 in case III where it was 150. Iterations and CPU timings on a $933 \mathrm{MHz}$ Pentium III are shown in Tab. 1 and Tab. 2 for each stage. Evidently, the computational cost would be considerably reduced using a massive parallel machine, where the GAs become competitive with other optimisation methods.

Finally, as example, Fig. 2 shows the wind field obtained by the model, in the second experiment, at a height of $200 \mathrm{~m}$ using the values of the parameters corresponding to Stage 6 . Here, the measures of the four stations have been taken into account for determining the interpolated wind field.

Table 1. First experiment corresponding to the case I analysed in [15]. Strategy of genetics algorithms, best evaluation of the fitness function and values of the parameters (fixed values are written between brackets)

\begin{tabular}{|c|c|c|}
\cline { 2 - 3 } \multicolumn{1}{c|}{} & Stage 1 & Stage 2 \\
\hline GAs strategy & BT, U, R & SU, U, G \\
\hline Iterations & 17 & 1 \\
\hline CPU time (s) & 1548 & 108 \\
\hline Best Fitness & 0.107 & 0.107 \\
\hline$\alpha$ & 9.810 & 9.727 \\
\hline$\varepsilon$ & 0.010 & 0.029 \\
\hline$\gamma$ & $(0.300)$ & 0.284 \\
\hline
\end{tabular}

Table 2. Second experiment corresponding to the case III analysed in [15]. Strategy of genetics algorithms, best evaluation of the fitness function and values of the parameters (fixed values are written between brackets)

\begin{tabular}{|c|c|c|c|c|c|c|}
\cline { 2 - 7 } \multicolumn{1}{c|}{} & Stage 1 & Stage 2 & Stage 3 & Stage 4 & Stage 5 & Stage 6 \\
\hline GAs strategy & SU, U, G & SU, U, R & SU, U, R & SU, U, R & SU, U, R & SU, U, R \\
\hline Iterations & 110 & 147 & 37 & 16 & 29 & 61 \\
\hline CPU time (s) & 8514 & 12120 & 2958 & 1362 & 2406 & 7050 \\
\hline Best Fitness & 0.234 & 0.227 & 0.222 & 0.223 & 0.222 & 0.222 \\
\hline$\alpha$ & 4.182 & 5.041 & $(5.041)$ & 4.765 & 5.699 & 6.080 \\
\hline$\varepsilon$ & 0.003 & 0.272 & 0.281 & $(0.281)$ & 0.292 & 0.282 \\
\hline$\gamma$ & $(0.300)$ & 0.490 & 0.493 & 0.498 & $(0.498)$ & 0.494 \\
\hline$\gamma^{\prime}$ & $(0.400)$ & $(0.400)$ & 0.154 & 0.153 & 0.154 & 0.153 \\
\hline
\end{tabular}




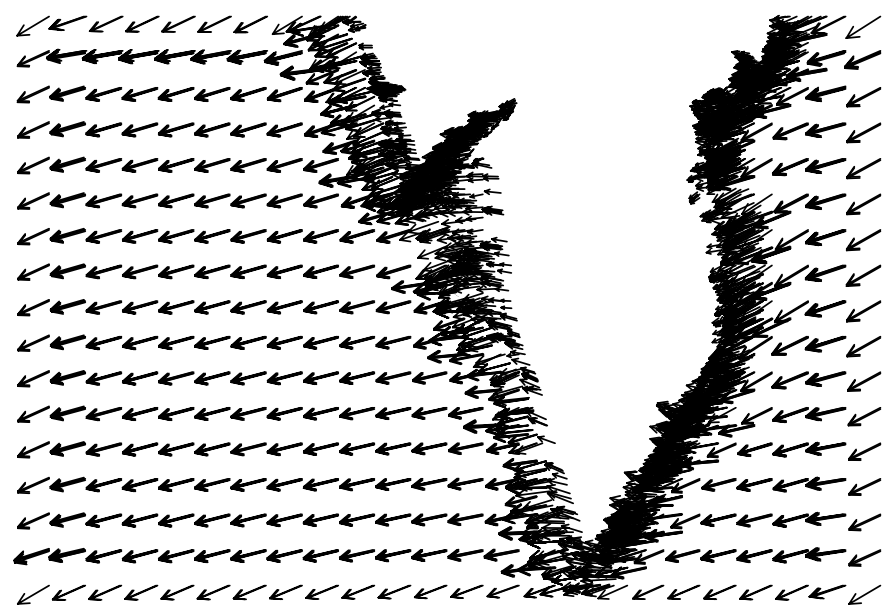

Fig. 2. Wind field solution related to the second experiment at a height of $200 \mathrm{~m}$

\section{Conclusions}

The estimation of several parameters is essential for the efficiency of a 3-D mass consistent model for wind field adjustment. The numerical experiments have shown that these codes are very sensitive to the values chosen for $\alpha, \varepsilon, \gamma$ and $\gamma^{\prime}$. A methodology for solving these parameter estimation problems is proposed. Genetic algorithms have proved to be an efficient and robust tool for these optimisation problems when several parameters are involved (see also [13]).

\section{References}

1. de Baas, A.F.: Scaling Parameters and their Estimation. In: Lalas, D.P., Ratto, C.F. (eds.): Modelling of Atmospheric Flow Fields. World Sci. Singapore (1996) 87-102

2. Barnard, J.C., Wegley, H.L., Hiester, T.R.: Improving the Performance of Mass Consistent Numerical Model Using Optimization Techniques. J. Climate Appl. Meteorol. 26 (1987) 675-686

3. Businger, J.A., Arya, S.P.S.: Heights of the Mixed Layer in the Stable, Stratified Planetary Boundary Layer. Adv. Geophys. 18A (1974) 73-92

4. Davis, C.G., Bunker, S.S., Mutschlecner, J.P.: Atmospheric Transport Models for Complex Terrain. J. Climate Appl. Meteorol. 23 (1984) 235-238

5. Davis, L.: Handbook of Genetic Algorithms. Van Nostrand Reinhold (1991)

6. Garratt, J.R.: Observations in the Nocturnal Boundary Layer. Boundary-Layer Meteorol. 22(1) (1982) 21-48 
7. Geai, P.: Methode d'Interpolation et de Reconstitution Tridimensionelle d'un Champ de Vent: le Code d'Analyse Objective MINERVE. Technical Report DER/HE/34-87.03, EDF, Chatou, France (1985)

8. Holland, J.: Adaption in Natural and Artificial Systems. MIT Press (1992)

9. Kitada, T., Kaki, A., Ueda, H., Peters, L.K.: Estimation of Vertical Air Motion from Limited Horizontal Wind Data - A Numerical Experiment. Atmos. Environ. 17 (1983) 2181-2192

10. Lalas, D.P., Tombrou, M., Petrakis, M.: Comparison of the Performance of Some Numerical Wind Energy Siting Codes in Rough Terrain. In European Community Wind Energy Conference, Herning, Denmark (1988)

11. Levine, D.: A Parallel Genetic Algorithm for the Set Partitioning Problem. Ph. D. Thesis, Illinois Institute of Technology / Argonne National Laboratory (1994)

12. Montenegro, R., Montero, G., Escobar, J.M., Rodríguez, E., González-Yuste, J.M.: Tetrahedral Mesh Generation for Environmental Problems over Complex Terrains. Lecture Notes in Computer Science. Springer Verlag, Berlin Heidelberg New York (2002). Submitted.

13. Montero, G.: Solving Optimal Control Problems by GAs. Nonlin. Anal., Theor., Meth., \& Appl. 30(5) (1997) 2891-2902

14. Montero, G., Montenegro, R., Escobar, J.M.: A 3-D Model for Wind Field Adjustment. J. Wind Eng. \& Ind. Aer. 74-76 (1998) 249-261

15. Montero, G., Sanín, N.: 3-D Modelling of Wind Field Adjustment Using Finite Differences in a Terrain Conformal Coordinate System. J. Wind Eng. \& Ind. Aer. 89 (2001) 471-488

16. Moussiopoulos, N., Flassak, Th., Knittel, G.: A Refined Diagnostic Wind Model. Environ. Software 3 (1988) 85-94

17. Panofsky, H.A., Dutton, J.A.: Atmospheric Turbulence. John Wiley, New York (1984)

18. Ratto, C.F.: An Overview of Mass-consistent Models. In: Lalas, D.P., Ratto, C.F. (eds.): Modelling of Atmospheric Flow Fields. World Sci. Singapore (1996) 379-400

19. Ratto, C.F.: The AIOLOS and WINDS Codes. In: Lalas, D.P., Ratto, C.F. (eds.): Modelling of Atmospheric Flow Fields. World Sci. Singapore (1996) 421-431

20. Ross, D.G., Smith, I.N., Manins, P.C., Fox, D.G.: Diagnostic Wind Field Modelling for Complex Terrain: Model Development and Testing. J. Appl. Meteorol. 27 (1988) 785-796

21. Sherman, C.A.: A Mass-Consistent Model for Wind Fields over Complex Terrain. J. Appl. Meteorol. 17 (1978) 312-319

22. Spears, W., DeJong, K.: On the Virtues of Parametrized Uniform Crossover. Proc. of the Fourth International Conference on Genetic Algorithms (1991)

23. Syswerda, G.: Uniform Crossover in Genetic Algorithms. Proc. of the Third International Conference on Genetic Algorithms (1989)

24. Tombrou, M., Lalas, D.P.: A Telescoping Procedure for Local Wind Energy Potential Assessment. In: Palz, W. (ed.): European Community Wind Energy Conference, H.S. Stephens \& Associates, Madrid (1990)

25. Whitley, D.: The GENITOR Algorithm and Selection Pressure: Why Rank-based Allocation of Reproductive Trials is Best. Proc. of the Third International Conference on Genetic Algorithms (1989)

26. Whitley, D.: GENITOR: A Different Genetic Algorithm. Rocky Mountain Conference on Artificial Intelligence (1988)

27. Winter, G., Montero, G., Ferragut, L., Montenegro, R.: Adaptive Strategies Using Standard and Mixed Finite Elements for Wind Field Adjustment. Solar Energy 54(1) (1995) 49-56 